Preprint typeset in JHEP style - HYPER VERSION

$\mathrm{IPPP} / 05 / 06$

$\mathrm{DCPT} / 05 / 12$

hep-ph/0503129

\title{
Regularization by Dimensional Reduction: Consistency, Quantum Action Principle, and Supersymmetry
}

\author{
Dominik Stöckinger \\ Institute for Particle Physics Phenomenology, Physics Department, University of \\ Durham, Durham DH1 3LE, UK \\ E-mail: Dominik.Stockinger@durham.ac.uk
}

\begin{abstract}
It is proven by explicit construction that regularization by dimensional reduction can be formulated in a mathematically consistent way. In this formulation the quantum action principle is shown to hold. This provides an intuitive and elegant relation between the $D$-dimensional Lagrangian and Ward or Slavnov-Taylor identities, and it can be used in particular to study to what extent dimensional reduction preserves supersymmetry. We give several examples of previously unchecked cases.
\end{abstract}

KEYwords: Renormalization, Regularization, Supersymmetry. 


\section{Contents}

1. Introduction 1

2. Consistent dimensional reduction 3

3. Quantum action principle 5

4. Supersymmetry of DRED 8

4.1 Previous checks 8

4.2 General breaking of the Slavnov-Taylor identity in DRED 9

4.3 Examples 11

5. Conclusions 14

A. Explicit construction of Q4S

\section{Introduction}

Regularization of loop contributions has been a notorious and long standing problem for supersymmetric gauge theories. Dimensional regularization (DREG) [1] and its variant dimensional reduction (DRED) [2] are by far the most common regularization schemes. However, both schemes have well-known serious shortcomings.

DREG explicitly breaks supersymmetry because the number of degrees of freedom of gauge bosons and gauginos does not match for $D \neq 4$. In practical calculations this breaking would have to be corrected by adding suitable supersymmetryrestoring counterterms [3,4], whose existence is always guaranteed by the renormalizability of supersymmetric gauge theories [5-7]. But since these counterterms do not originate from multiplicative renormalization their evaluation and implementation poses a significant practical complication.

In DRED only momenta are treated in $D$ dimensions whereas $\gamma$-matrices and gauge fields remain ordinary 4-vectors, and thus the direct supersymmetry breaking of DREG is avoided. Hence DRED is used in most practical cases where the supersymmetry breaking of DREG would matter. However, DRED is mathematically inconsistent [8]. In [8] it has been shown that the combination of 4dimensional $\gamma$-matrices and $\epsilon$-tensors with a $D$-dimensional $g^{\mu \nu}$ leads to the relation 
$0=D(D-1)(D-2)(D-3)(D-4)$, which is obviously inconsistent with non-integer values of $D$.

This problem of regularization is not only a curiosity of supersymmetric field theories. It is of increasing practical importance as more and more predictions have to be evaluated at the loop-level in order to match the current or expected future experimental accuracy. For example, for observables such as the lightest Higgs boson mass, $(g-2)$ of the muon, or electroweak precision observables even two-loop corrections have to be known [9]. The need for a better understanding of DRED and the related $\overline{D R}$ scheme has also been emphasized by the SPA "Supersymmetry Parameter Analysis" Project [10].

Recently, interest in properties of DRED has increased also because of the mass factorization problem observed in Ref. [11] and emphasized again in Ref. [12]. However, in the context of supersymmetry DRED is most useful for loop diagrams, while a factorization problem has only been found for real gluon radiation diagrams. Moreover, many important calculations such as the ones reviewed in Ref. [9] or the evaluation of $\beta$-functions [13] do not rely on factorization at all. In the following we will focus on the first problem of mathematical inconsistency and related questions.

Of course, the mathematical inconsistency should not matter in many practical cases [14]. However, this inconsistency (which implies that one initial expression can lead to different results depending on the calculational steps) makes it very hard to prove generally valid statements on DRED.

Most importantly, even in DRED it is unclear whether or to what extent supersymmetry is actually preserved. It is only known that some supersymmetry relations are satisfied at the one- or two-loop level, but even at the one-loop level the checks do not exhaust all Green functions that could be affected by a supersymmetry breaking.

In this paper we show the validity of two statements:

1. DRED can be formulated in a mathematically consistent way (Section 2). The consistent formulation is essentially identical to the way DRED is usually applied, with the exception that no Fierz identities can be used. We give an explicit representation of all formally $D$-dimensional quantities in terms of mathematically well-defined objects. This ensures consistency of the calculational prescriptions, and uniqueness and existence of the results.

2. In this formulation of DRED, the quantum action principle is valid (Section 3). This theorem provides a direct relation between the regularized Lagrangian $\mathcal{L}$ and Ward or Slavnov-Taylor identities for Green functions at all orders:

$$
i \delta\left\langle T \phi_{1} \ldots \phi_{n}\right\rangle=\left\langle T \phi_{1} \ldots \phi_{n} \Delta\right\rangle
$$

where $\delta=\delta \phi_{i} \frac{\delta}{\delta \phi_{i}}$ denotes some symmetry transformation and $\Delta=\int d^{D} x \delta \mathcal{L}$ (see Sec. 3 for further variants of the theorem). Thus this relation constitutes a simple and general tool to study the symmetry-properties of DRED. 
In a third step the quantum action principle is applied to supersymmetry transformations (Section đ). Supersymmetry Ward or Slavnov-Taylor identities can be brought into the form

$$
\delta_{\mathrm{SUSY}}\left\langle T \phi_{1} \ldots \phi_{n}\right\rangle=0
$$

Hence if $\delta_{\text {SUSY }} \mathcal{L}$ were zero, the quantum action principle would immediately show the validity of supersymmetry Ward and Slavnov-Taylor identities to all orders.

However, it turns out that $\delta_{\mathrm{SUSY}} \mathcal{L} \neq 0$ in consistent DRED because in order to avoid the mathematical inconsistency a "quasi-4-dimensional" space (Q4S) [15] is introduced, where Fierz identities do not hold. Correspondingly, the supersymmetry identities (1.2) are not generally valid but only to the extent that the insertion of $\delta_{\text {SUSY }} \mathcal{L}$ does not contribute on the right-hand side of eq. (1.1).

It is often dramatically simpler to prove that the right-hand side of (1.1) vanishes than to evaluate the left-hand side of (1.2). Hence by using the quantum action principle many more Ward or Slavnov-Taylor identities are amenable to checks, and the checks themselves are drastically simplified. In Sec. 团 we will apply this strategy and check several identities of practical interest at the one- and two-loop level.

\section{Consistent dimensional reduction}

In DRED, momenta are continued from 4 to $D$ dimensions, while gauge fields and $\gamma$-matrices remain 4 -dimensional objects. The $D$-dimensional momenta live in a $D$ dimensional space with metric tensor $\hat{g}^{\mu \nu}$, and the 4-dimensional quantities live in a 4-dimensional space with metric tensor $g^{\mu \nu}$. It is important that the relation

$$
\not p \not p=\frac{1}{2} p_{\mu} p_{\nu}\left\{\gamma^{\mu}, \gamma^{\nu}\right\}=p_{\mu} p_{\nu} g^{\mu \nu}=p^{2}
$$

holds for $D$-dimensional momenta such that e.g. a Dirac propagator $(\not p+m) /\left(p^{2}-m^{2}\right)$ is indeed the inverse of $(\not p-m)$. This requires that the $D$-dimensional space is a subspace of the 4-dimensional one and one has the projector relations

$$
g^{\mu \nu} \hat{g}_{\nu}{ }^{\rho}=\hat{g}^{\mu \rho} .
$$

For ordinary DREG, it is well-known that the $D$-dimensional space can be realized only formally. Actually it is an infinite dimensional vector space on which $\hat{g}^{\mu \nu}$ is defined as a certain operator with all desired properties that resemble $D$-dimensional behaviour $[16,17]$. Therefore it seems difficult for DRED to realize such a formally $D$-dimensional, but actually infinite dimensional space that is at the same time a subspace of the 4-dimensional space. Indeed it is eq. (2.2), combined with several purely 4 -dimensional relations between the $\epsilon$-tensor and $g^{\mu \nu}$ that leads to the inconsistency found in [8].

The solution is to realize the 4-dimensional space as a "quasi-4-dimensional" space Q4S that retains essential 4-dimensional properties but is in fact also infinite 
dimensional. Such a space was already postulated in [15], and in App. A it is shown that it is indeed possible to construct a hierarchy of two infinite dimensional spaces $\mathrm{Q} D \mathrm{~S} \subset \mathrm{Q} 4 \mathrm{~S}$ such that $\mathrm{Q} D \mathrm{~S}$ is formally D-dimensional, Q4S formally 4-dimensional and $\mathrm{Q} D \mathrm{~S}$ is a subspace of $\mathrm{Q} 4 \mathrm{~S}$. The complement of the $D$-dimensional space is an $\epsilon=4-D$-dimensional space $\mathrm{Q} \epsilon \mathrm{S}, \mathrm{Q} 4 \mathrm{~S}=\mathrm{Q} D \mathrm{~S} \oplus \mathrm{Q} \epsilon \mathrm{S}$.

In App. A we also give an explicit construction of all objects in these spaces that are needed for field theoretical calculations. This explicit construction guarantees that all calculational rules are consistent and that all calculations lead to unambiguous and well-defined results. In the following we list the properties and calculational rules of these objects, the metric tensors, $\gamma$-matrices, $\gamma_{5}$, and of charge conjugation.

On Q4S, QDS and Q $\epsilon \mathrm{S}$, metric tensors $g^{\mu \nu}, \hat{g}^{\mu \nu}$ and $\tilde{g}^{\mu \nu}$ can be defined that satisfy the following relations:

$$
\begin{aligned}
g^{\mu \nu} & =\hat{g}^{\mu \nu}+\tilde{g}^{\mu \nu} & & g^{\mu \nu} g_{\mu \nu}=4 \\
g^{\mu \nu} \hat{g}_{\nu}{ }^{\rho} & =\hat{g}^{\mu \rho} & & \hat{g}^{\mu \nu} \hat{g}_{\mu \nu}=D \\
g^{\mu \nu} \tilde{g}_{\nu}{ }^{\rho} & =\tilde{g}^{\mu \rho} & & \tilde{g}^{\mu \nu} \tilde{g}_{\mu \nu}=\epsilon=4-D \\
\hat{g}^{\mu \nu} \tilde{g}_{\nu}{ }^{\rho} & =0 & &
\end{aligned}
$$

In practical calculations it is sufficient to know these relations. The only practical consequence of the infinite dimensional nature of the underlying spaces is that index counting is not possible: E.g. out of five Lorentz indices $\mu_{1}, \mu_{2}, \mu_{3}, \mu_{4}, \mu_{5}$ two must be equal in truly four dimensions, but in Q4S all of them can be different.

In $\mathrm{Q} 4 \mathrm{~S}, \gamma$-matrices can be defined that satisfy the Dirac algebra

$$
\left\{\gamma^{\mu}, \gamma^{\nu}\right\}=2 g^{\mu \nu}
$$

and have the hermiticity property

$$
\left(\gamma^{\mu}\right)^{\dagger}=\gamma^{0} \gamma^{\mu} \gamma^{0}
$$

Again these relations resemble 4-dimensional behaviour and are sufficient in practice, but as a consequence of Q4S further relations like Fierz identities are not valid.

Concerning the definition of $\gamma_{5}$, there are two options. Either we define it as in 4 dimensions and as in the HVBM-scheme $[1,18]$ as $\bar{\gamma}_{5}=i \gamma^{0} \gamma^{1} \gamma^{2} \gamma^{3}$, or we define $\gamma_{5}$ as totally anticommuting (the existence of such an object that anticommutes with all infinitely many $\gamma^{\mu}$ is shown in App. A). To distinguish the two options we denote the purely 4-dimensional one by $\bar{\gamma}_{5}$. If the second option is used, we have

$$
\left\{\gamma_{5}, \gamma^{\mu}\right\}=0
$$

and this is the way DRED is usually defined. Hence the results we will obtain for this case will immediately hold for all applications of DRED that rely on eqs. (2.3,2.4,2.6). The drawback of this option is that traces of $\gamma_{5}$ and four or more $\gamma$-matrices vanish in 
contrast to the 4-dimensional case. ${ }^{1}$ In this respect DRED is not better than DREG with anticommuting $\gamma_{5}$. For the option of $\bar{\gamma}_{5}$ it is useful to introduce additional projectors onto the true 4-dimensional space, and its complement, $\bar{g}^{\mu \nu}, \tilde{\tilde{g}}^{\mu \nu}$ :

$$
\begin{aligned}
g^{\mu \nu} & =\bar{g}^{\mu \nu}+\tilde{\tilde{g}}^{\mu \nu} & & \bar{g}^{\mu \nu} \tilde{\tilde{g}}_{\nu}{ }^{\rho}=0 \\
g^{\mu \nu} \bar{g}_{\nu}{ }^{\rho} & =\bar{g}^{\mu \rho} & & \bar{g}^{\mu \nu} \bar{g}_{\mu \nu}=4 \\
g^{\mu \nu} \tilde{\tilde{g}}_{\nu}{ }^{\rho} & =\tilde{\tilde{g}}^{\mu \rho} & & \tilde{\tilde{g}}^{\mu \nu} \tilde{\tilde{g}}_{\mu \nu}=0
\end{aligned}
$$

Using the notation $\tilde{\tilde{a}}^{\mu}=\tilde{\tilde{g}}^{\mu}{ }_{\nu} a^{\nu}$, we then have the anticommutation relation

$$
\left\{\bar{\gamma}_{5}, \gamma^{\mu}\right\}=2 \bar{\gamma}_{5} \tilde{\tilde{\gamma}}^{\mu}
$$

Both definitions of $\gamma_{5}$ have the additional properties

$$
\begin{array}{ll}
\gamma_{5}^{\dagger}=\gamma_{5} & \bar{\gamma}_{5}^{\dagger}=\bar{\gamma}_{5} \\
\gamma_{5}^{2}=1 & \bar{\gamma}_{5}^{2}=1
\end{array}
$$

Particularly for calculations in supersymmetric models, it is very important to use charge conjugation in order to simplify products of spinors and $\gamma$-matrices. In App. A the charge conjugation matrix $C$ is constructed in Q4S, showing that it is possible to use all the usual charge conjugation relations even in Q4S. Charge conjugated spinors $\psi^{C}$ can be defined and bilinear expressions can be rewritten in the following way:

$$
\begin{aligned}
\psi^{C} & =-i \gamma^{0} C \psi^{*} & \overline{\psi_{1}^{C}} & =-i \psi^{T} C \\
\bar{\psi}_{1} \Gamma \psi_{2} & =\overline{\psi_{2}^{C}} \Gamma^{C} \psi_{1}^{C} & \text { with } \Gamma^{C} & =-C \Gamma^{T} C
\end{aligned}
$$

In the rearrangement formula for $\bar{\psi}_{1} \Gamma \psi_{2}$, anticommuting spinors have been assumed. If $\psi_{1,2}$ commute, there is an additional minus sign on the right-hand side. The results for $\Gamma^{C}$ are

$$
\left\{1, \gamma_{5}, \bar{\gamma}_{5}, \gamma^{\mu}, \gamma^{\mu} \gamma_{5}, \gamma^{\mu} \bar{\gamma}_{5}\right\}^{C}=\left\{1, \gamma_{5}, \bar{\gamma}_{5},-\gamma^{\mu},-\gamma_{5} \gamma^{\mu},-\bar{\gamma}_{5} \gamma^{\mu}\right\}
$$

\section{Quantum action principle}

The quantum action principle (1.1) is a simple relation between the symmetry properties of the regularized Lagrangian and the full Green functions. Heuristically, it can be derived in the following way using the functional integral

$$
Z(J)=\int \mathcal{D} \phi e^{i \int d^{D} x(\mathcal{L}+J \phi)},
$$

\footnotetext{
${ }^{1}$ The proof is analogous to the DREG-case if we write $\gamma^{\mu}=\hat{\gamma}^{\mu}+\tilde{\gamma}^{\mu}$ with $\hat{\gamma}^{\mu} \hat{\gamma}_{\mu}=D, \tilde{\gamma}^{\mu} \tilde{\gamma}_{\mu}=4-D$. Starting e.g. from $\operatorname{Tr}\left(\hat{\gamma}^{\alpha} \hat{\gamma}_{\alpha} \gamma_{5} \hat{\gamma}^{\mu} \hat{\gamma}^{\nu} \hat{\gamma}^{\rho} \hat{\gamma}^{\sigma}\right)$ one obtains $(4-D) \operatorname{Tr}\left(\gamma_{5} \hat{\gamma}^{\mu} \hat{\gamma}^{\nu} \hat{\gamma}^{\rho} \hat{\gamma}^{\sigma}\right)=0$. Similarly one obtains $(3-D) \operatorname{Tr}\left(\gamma_{5} \tilde{\gamma}^{\mu} \hat{\gamma}^{\nu} \hat{\gamma}^{\rho} \hat{\gamma}^{\sigma}\right)=0$, and so on. Finally one can show in this way that all traces of the form $\operatorname{Tr}\left(\gamma_{5}\left(\operatorname{arbitrary}\right.\right.$ number of $\left.\left.\gamma^{\mu_{i}}\right)\right)$ vanish if $D$ is non-integer.
} 
where a symbolic notation $\phi$ for all quantum fields and $J$ for all corresponding sources is used. Performing a variable transformation

$$
\phi \rightarrow \phi+\delta \phi
$$

in the functional integral and expanding to first order in $\delta$ yields

$$
0=\int \mathcal{D} \phi\left(\int d^{D} x i(\delta \mathcal{L}+J \delta \phi)\right) e^{i \int d^{D} x(\mathcal{L}+J \phi)} .
$$

This equation corresponds exactly to eq. (1.1) for Green functions. In addition to transformations of quantum fields, often variations of external fields or parameters are considered. For such cases similar relations hold. In summary, the quantum action principle has three variants:

- Variation of quantum fields: $\delta=\int d^{D} x \delta \phi_{i}(x) \frac{\delta}{\delta \phi_{i}(x)}$.

$$
i \delta\left\langle T \phi_{1} \ldots \phi_{n}\right\rangle=\left\langle T \phi_{1} \ldots \phi_{n} \Delta\right\rangle
$$

where $\Delta=\int d^{D} x \delta \mathcal{L}$ and $\delta$ has to be pulled into the brackets on the left-hand side.

- Variation of an external (non-propagating) field $Y(x)$ :

$$
-i \frac{\delta}{\delta Y(x)}\left\langle T \phi_{1} \ldots \phi_{n}\right\rangle=\left\langle T \phi_{1} \ldots \phi_{n} \Delta\right\rangle
$$

with $\Delta=\frac{\delta}{\delta Y(x)} \int d^{D} x \mathcal{L}$.

- Variation of a parameter $\lambda$ :

$$
-i \frac{\partial}{\partial \lambda}\left\langle T \phi_{1} \ldots \phi_{n}\right\rangle=\left\langle T \phi_{1} \ldots \phi_{n} \Delta\right\rangle
$$

with $\Delta=\frac{\partial}{\partial \lambda} \int d^{D} x \mathcal{L}$.

The two other variants can be similarly derived from the functional integral, and the different signs can be easily understood in this way.

However, the derivation from the functional integral is only heuristic because the measure $\mathcal{D} \phi$ has tacitly been assumed to be invariant under the variable transformation. But this is precisely the point where the regularization enters. Hence the quantum action principle has to be established separately for each regularization.

For BPHZ-renormalization, it has been proven in [19], and for DREG, it has been proven in [18]. The proofs for the three variants are very similar, so we restrict ourselves here to the first one. We will see that the proof for DREG can be applied with no essential modification also to DRED. A crucial prerequisite however is the mathematical consistency of DRED as formulated in Sec. 2. 
Partly, the proof of eq. (1.1) is pure combinatorics of Feynman diagrams, and this part is independent of the regularization and not discussed here. The more intricate part of the proof is related to the fact that Feynman diagrams treat the kinetic part of the Lagrangian different from the interaction part. Decomposing $\mathcal{L}+J \phi=\mathcal{L}_{0}+\mathcal{L}_{\text {int }}$, where $\mathcal{L}_{0}$ contains the kinetic terms determining the propagators and $\mathcal{L}_{\text {int }}$ contains the interaction and source terms, the generating functional $Z(J)$ has the following expression in terms of Feynman diagrams:

$$
Z(J)=\left\langle T \exp \left(i \int d^{D} x \mathcal{L}_{\mathrm{int}}\right)\right\rangle
$$

This expression has to be evaluated with free fields using Wick's theorem to obtain the contractions giving rise to the propagators. Similarly, the Feynman diagram identity corresponding to eq. (1.1) is given by

$$
0=\left\langle T\left(\int d^{D} x\left(\delta \mathcal{L}_{0}+\delta \mathcal{L}_{\text {int }}\right)\right) \exp \left(i \int d^{D} x \mathcal{L}_{\text {int }}\right)\right\rangle .
$$

Hence in order to prove this identity one has to show that the terms involving $\delta \mathcal{L}_{0}$ cancel the ones involving $\delta \mathcal{L}_{\text {int }}$. Writing $\mathcal{L}_{0}=\frac{1}{2} \phi_{i} D_{i j} \phi_{j}$ with some differential operator $D_{i j}$, the terms involving $\delta \mathcal{L}_{0}=\delta \phi_{i} D_{i j} \phi_{j}$ result in two different kinds of Wick contractions involving $\phi_{j}$ :

$$
\begin{aligned}
& \left\langle\left(\int d_{\uparrow}^{D} x \delta \phi_{i} D_{i j} \phi_{j}\right)\left(i \int d^{D} x \mathcal{L}_{\text {int }}\right) \ldots\left(i \int d^{D} x \mathcal{L}_{\text {int }}\right)\right\rangle \\
& +\left\langle\left(\int d^{D} x \delta \phi_{i} D_{i j} \phi_{\uparrow}\right)\left(i \int d^{D} x \underset{\uparrow}{\mathcal{L}_{\mathrm{int}}}\right)\left(i \int d^{D} x \mathcal{L}_{\mathrm{int}}\right) \ldots\left(i \int d^{D} x \mathcal{L}_{\mathrm{int}}\right)\right\rangle,
\end{aligned}
$$

where the Wick contractions involving $\phi_{j}$ are denoted by arrows (Wick contractions of all other fields are not made explicit here). In momentum space Feynman diagrams, the first line always produces a loop integral over $D_{i j}$ times the propagator $P_{j k}$ from $\phi_{j}$ to some field $\phi_{k}$ within the composite operator $\delta \phi_{i}$. Now one crucial property of DRED and DREG is relevant, namely

$$
D_{i j} P_{j k}=i \delta_{i k}
$$

in momentum space even on the regularized level. Hence the loop integral corresponding to the first contraction in (3.9) is scaleless and therefore zero.

Contracting $\phi_{j}$ in the second line of (3.9) with some field $\phi_{k}$ within $\mathcal{L}_{\text {int }}$ results in the product $P_{j k} \frac{\delta \mathcal{L}_{\text {int }}}{\delta \phi_{k}}$. Using eq. (3.10) again, we find that eq. (3.9) becomes

$$
i^{2}\left\langle\left(\int d^{D} x \delta \phi_{i} \frac{\delta \mathcal{L}_{\text {int }}}{\delta \phi_{i}}\right)\left(i \int d^{D} x \mathcal{L}_{\text {int }}\right) \ldots\left(i \int d^{D} x \mathcal{L}_{\text {int }}\right)\right\rangle .
$$

Using this result in eq. (3.8) together with combinatorial arguments shows that the terms involving $\delta \mathcal{L}_{0}$ indeed cancel the ones involving $\delta \mathcal{L}_{\text {int }}$, proving the quantum action principle. 
To summarize, the two main points where the properties of DRED (shared by DREG) enter are eq. (3.10) and the fact that scaleless integrals vanish in dimensional schemes. In [18], eq. (3.10) has been proven not only in momentum space but also using the $\alpha$-representation for the propagators, where the relation is less obvious. The same proof is also valid in DRED without changes. However it relies on the fact that the expression $(\not p+m) /\left(p^{2}-m^{2}\right)$ for a Dirac propagator is really the inverse of the kinetic operator $(\not p-m)$, even if $p$ is $D$-dimensional and the $\gamma$-matrices are defined in $\mathrm{Q} 4 \mathrm{~S}$. The importance of this fact and its relation to the mathematical inconsistency of DRED with truly 4-dimensional $\gamma$-matrices has already been stressed in Sec. 2 .

\section{Supersymmetry of DRED}

In this section we apply the quantum action principle to obtain more information on the supersymmetry properties of DRED. What is known so far is limited to many one-loop cases and few cases beyond one-loop order, and the methods used up to now are not very easy to apply to complicated situations since they involve the evaluation of full Green functions including the finite parts. The application of the quantum action principle should lead to a significant simplification, and we will illustrate this with several non-trivial examples. We will start however with a brief overview of the current status (see also the review [14]).

\subsection{Previous checks}

Immediately after the invention of DRED [2], first checks of Ward identities were performed in [20]. The checks comprised several gauge Ward identities up to the twoloop level and supersymmetry Ward identities at the one-loop level. However, only supersymmetry Ward identities for propagators but not for 3- or 4-point functions were considered. In [15] the quantum action principle was assumed and it was argued that the propagator Ward identity in a supersymmetric gauge theory without matter fields should hold up to the three-loop level. In [3] an S-matrix identity connecting the gluino-squark-quark 3-point function and gauge interactions was checked at the one-loop level.

Ref. [21] demonstrated that supersymmetry Ward identities are not sufficient to describe the symmetry content of supersymmetric gauge theories if (as done in virtually all practical cases) the Wess-Zumino gauge is used. Instead, supersymmetry Slavnov-Taylor identities [5-7] constitute an exact representation of the symmetry content of these theories. They are the center of proofs of the absence of anomalies and the multiplicative renormalizability of supersymmetric gauge theories. In Refs. [4] several Slavnov-Taylor identities relating propagators, 3-point functions, and Green functions corresponding to loop-corrected supersymmetry transformations have been checked. 
In all checked cases, the respective identities turned out to be valid in DRED on the regularized level. However, it is noteworthy that even at the one-loop level there are Green functions such as four-scalar interactions that do not appear in any of the checked identities and hence could in principle still violate supersymmetry in DRED. In addition to the direct checks, in several cases DRED-results for $\beta$-functions of supersymmetric parameters could be checked either for internal consistency or against non-perturbative results [22].

\subsection{General breaking of the Slavnov-Taylor identity in DRED}

The present situation of DRED and supersymmetry is not completely satisfactory: So far DRED has passed all checks and there are many reasons to believe that DRED has an even wider range of validity, but there is no general proof or analysis whether or to what extent DRED really preserves supersymmetry. The quantum action principle is a suitable tool to perform such a general analysis. In the following we show how it can be applied to study supersymmetry Slavnov-Taylor identities in DRED.

The Slavnov-Taylor identities can be combined into a single equation for the generating functional $\Gamma$ for the one-particle irreducible (1PI) vertex functions:

$$
S(\Gamma)=0
$$

with

$$
S(\Gamma)=\int d^{D} x\left(\sum_{i} \frac{\delta \Gamma}{\delta Y_{i}(x)} \frac{\delta \Gamma}{\delta \varphi_{i}(x)}+\sum_{j} s \varphi_{j}^{\prime} \frac{\delta \Gamma}{\delta \varphi_{j}^{\prime}}\right) .
$$

Here $\varphi_{i}$ denote the quantum fields with non-linear BRS transformations, $Y_{i}$ the corresponding external fields that couple to these BRS transformations, and $\varphi_{j}^{\prime}$ denote the fields with linear BRS transformations $s \varphi_{j}^{\prime}$ (see Refs. [5-7] for further details). Eq. (4.1) describes the full symmetry content, i.e. gauge invariance, supersymmetry and translational invariance, and it should hold for the renormalized vertex functional $\Gamma$. The interesting question is whether it already holds for $\Gamma^{\mathrm{DRED}}$, the regularized vertex functional in DRED where no counterterms have been added.

By applying the usual Legendre transformations, we can apply the quantum action principle to the one-particle irreducible vertex functions and obtain

$$
S\left(\Gamma^{\mathrm{DRED}}\right)=i\left[S\left(\Gamma_{\mathrm{cl}}\right)\right] \cdot \Gamma^{\mathrm{DRED}},
$$

where $[X] \cdot \Gamma^{\text {DRED }}$ denotes the insertion of an operator $X$ into the 1 PI vertex functions analogous to the insertion on the right-hand side of eq. (1.1). $\Gamma_{\mathrm{cl}}$ denotes the treelevel contribution which is nothing but the classical action $\int d^{D} x \mathcal{L}$.

Eq. (4.3) can be used as the master equation for the study of symmetry properties of DRED. In order to make use of it, we will evaluate $S\left(\Gamma_{\mathrm{cl}}\right)$ for a general 
supersymmetric gauge theory. For simplicity we assume a simple gauge group with gauge field $A_{a}^{\mu}$, gaugino $\tilde{g}_{a}$, and chiral matter multiplets $\left(\phi_{i}, P_{L} \psi_{i}\right)$ with the projector $P_{L}=\frac{1}{2}\left(1-\gamma_{5}\right)$ and the gauge group generators $T_{i j}^{a}$. We use the general conventions of Ref. [7], rewritten in terms of 4 -spinors in order to make use of the calculational rules introduced in Sec. 2. In particular, the supersymmetry ghost is a commuting Majorana spinor $\epsilon=\epsilon^{C}$, the Faddeev-Popov ghost is denoted as $c_{a}$ and the sources for the BRS transformations $s \varphi_{i}$ generically as $Y_{\varphi_{i}}$. In the following formulas we write $\tilde{g}_{i j}=\tilde{g}_{a} T_{i j}^{a}$ and suppress indices wherever possible. Using the anticommuting version of $\gamma_{5}$, we obtain

$$
\begin{aligned}
S\left(\Gamma_{\mathrm{cl}}\right)= & \int d^{D} x\left[\Delta_{\text {gauge }}+\Delta_{\text {fix }}+\Delta_{\text {matter }}+\Delta_{Y_{\tilde{g}}}+\Delta_{Y_{c}}+\left(\Delta_{Y_{\psi}}+h . c .\right)\right] \\
\Delta_{\text {gauge }}= & \frac{i}{2} g f_{a b c}\left(\bar{\epsilon} \gamma^{\mu} \tilde{g}_{a}\right)\left(\overline{g_{g}} \gamma_{\mu} \tilde{g}_{c}\right) \\
\Delta_{\text {fix }}= & 0 \\
\Delta_{\text {matter }}= & -g\left[2\left(\bar{\psi} P_{R} \epsilon\right)\left(\overline{\tilde{g}} P_{L} \psi\right)+2\left(\bar{\epsilon} P_{L} \psi_{j}\right)\left(\overline{\psi_{i}} P_{R} \tilde{g}_{i j}\right)+\left(\overline{\psi_{i}} \gamma^{\mu} P_{L} \psi_{j}\right)\left(\bar{\epsilon} \gamma_{\mu} \tilde{g}_{i j}\right)\right] \\
\Delta_{Y_{\tilde{g}}}= & -\left(\overline{Y_{\tilde{g}}} \sigma^{\mu \nu} \epsilon\right)\left(D_{\mu} \bar{\epsilon} \gamma_{\nu} \tilde{g}_{a}\right)-i\left(\bar{\epsilon} \gamma^{\mu} \epsilon\right)\left(\overline{Y_{\tilde{g}_{a}}} D_{\mu} \tilde{g}_{a}\right)-i\left(\bar{\epsilon} \gamma_{5} \gamma^{\mu} D_{\mu} \tilde{g}_{a}\right)\left(\overline{Y_{\tilde{g}_{a}}} \gamma_{5} \epsilon\right), \\
\Delta_{Y_{c}}= & i Y_{c_{a}}\left(\bar{\epsilon} \gamma^{\mu} \epsilon\right)\left(\bar{\epsilon} \gamma_{\mu} \tilde{g}_{a}\right) \\
\Delta_{Y_{\psi}}= & -\sqrt{2} g\left[2\left(\overline{Y_{\psi}} P_{L} \epsilon\right)\left(\bar{\epsilon} P_{R} \tilde{g}\right) \phi+\left(\bar{\epsilon} \gamma^{\mu} \tilde{g}_{i j}\right)\left(\overline{Y_{\psi_{i}}} P_{L} \gamma_{\mu} \epsilon\right) \phi_{j}\right] \\
& +2 i\left[\left(\overline{Y_{\psi}} P_{L} \epsilon\right) \bar{\epsilon} \gamma^{\mu}+\left(\overline{Y_{\psi}} P_{L} \gamma^{\mu} \epsilon\right) \bar{\epsilon}-\frac{1}{2}\left(\bar{\epsilon} \gamma^{\mu} \epsilon\right) \overline{Y_{\psi}}\right] D_{\mu} P_{L} \psi
\end{aligned}
$$

All these terms vanish identically in four dimensions where Fierz rearrangements are possible. The term $\Delta_{\text {gauge }}$ was already obtained in [15] in the discussion of supersymmetry Ward identities. As will be shown in the next subsection, the insertion of these terms into diagrams as in (4.3) still vanishes in many cases, which explains the fact that no supersymmetry-violation of DRED has been found so far.

If the strictly 4-dimensional option $\bar{\gamma}_{5}$ is used in the definition of the regularized Lagrangian, the regularized theory and the breaking $S\left(\Gamma_{\mathrm{cl}}\right)$ is modified. For example, $\Delta_{\text {fix }}$ and $\Delta_{\text {matter }}$ contain the additional terms

$$
\bar{c}_{a} \bar{\epsilon} \tilde{\tilde{\gamma}}_{\mu} \bar{\gamma}_{5} \epsilon \partial^{\mu} D_{a}-\sqrt{2}\left[\phi^{\dagger} \bar{\epsilon} D \tilde{\tilde{D}} P_{L} \psi+\bar{\psi} P_{R} \tilde{\tilde{D}} \not D \epsilon \phi-i F^{\dagger} \overline{\tilde{\epsilon}} \tilde{\tilde{D}} P_{L} \psi-i \bar{\psi} P_{R} \tilde{\tilde{D}} \epsilon F\right]
$$

where $D_{a}=-g \phi^{\dagger} T^{a} \phi$ and $F_{i}^{\dagger}=-\partial W / \partial \phi_{i}$ with the superpotential $W$. It can be easily seen that inserting these terms into the simplest Green functions such as $\left\langle T \phi^{\dagger} \psi \Delta_{\text {matter }}\right\rangle$ leads to non-vanishing contributions already at the one-loop level. Hence if $\bar{\gamma}_{5}$ is used instead of $\gamma_{5}$, even the simplest Slavnov-Taylor identities relating one-loop propagators are violated, just like in DREG. Therefore and since in all practical applications of DRED the fully anticommuting $\gamma_{5}$ is used we will restrict ourselves to this case in the following. 


\subsection{Examples}

Now we use the master equation (4.3) and the explicit result (4.4) for the breaking to study the supersymmetry properties of DRED. We focus on three examples of practical interest but will exhibit also several general features. The examples are ${ }^{2}$

- The Slavnov-Taylor identity relating the $\phi$ and $\psi$ propagators $\Gamma_{\phi^{\dagger} \phi}$ and $\Gamma_{\psi \bar{\psi}}$. This identity is derived from the derivative $\frac{\delta^{3} S(\Gamma)}{\delta \phi^{\dagger} \delta \psi \delta \bar{\epsilon}}=0$ of (4.1) and reads

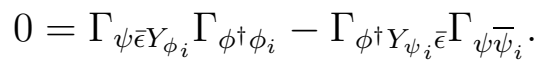

This identity expresses in particular the mass degeneracy of the bosonic and fermionic components of a chiral multiplet and is obviously one of the most fundamental supersymmetry relations.

- The Slavnov-Taylor identity relating the loop-corrected supersymmetry transformations of $\phi$ and $\psi, \Gamma_{Y_{\phi} \psi \bar{\epsilon}}$ and $\Gamma_{\phi \epsilon} \bar{Y}_{\psi}$. It is derived from $\frac{\delta^{4} S(\Gamma)}{\delta \phi \delta \epsilon \delta \bar{\epsilon} \delta Y_{\phi}}=0$ and reads

$$
0=\Gamma_{Y_{\phi} \psi_{i} \bar{\epsilon}} \Gamma_{\phi \epsilon \bar{Y}_{\psi_{i}}}-\Gamma_{\phi Y_{\psi}{ }_{i}{ }_{i} \Gamma^{-}} \Gamma_{Y_{\phi} \epsilon \bar{\psi}_{i}^{C}}+2 \not p+\ldots
$$

where $p$ is the momentum flowing into $\phi$ and the dots denote terms involving power-counting finite Green functions that vanish at tree-level and do not receive counterterm contributions. The appearance of loop corrections to the supersymmetry transformations is discussed in $[4,23]$. The above identity expresses the fact that the loop-corrected supersymmetry transformations still have to be in agreement with the supersymmetry algebra $\{Q, \bar{Q}\}=2 \not p$.

- The Slavnov-Taylor identity determining the $\phi^{4}$ interaction. In supersymmetric models the coupling constant of a $\phi^{4}$ interaction is never a free parameter but it can be related to gauge and superpotential couplings. For example, in the Minimal Supersymmetric Standard Model, the quartic Higgs boson self coupling can be entirely expressed in terms of the gauge coupling. This is of paramount importance for phenomenology since it leads to the tree-level prediction $M_{h}<M_{Z}$ and to strong constraints on the Higgs boson mass $M_{h}$ even at the loop level (see [9] and references therein). The corresponding SlavnovTaylor identity can be derived from $\frac{\delta^{5} S(\Gamma)}{\delta \phi^{\dagger} \delta \phi \delta \phi^{\dagger} \delta \psi \delta \bar{\epsilon}}=0$ and reads

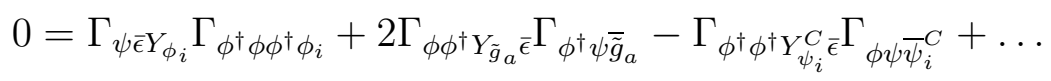

It relates $\Gamma_{\phi^{\dagger} \phi \phi^{\dagger} \phi_{i}}$ to a gauge interaction (second term) and a superpotential interaction (third term). Such an identity has not been considered in the literature so far.

\footnotetext{
${ }^{2}$ In order not to overload the following formulas, we suppress all indices that are not summed over. Furthermore, we always write just $\psi$ instead of $P_{L} \psi$ for the fermionic components of chiral multiplets.
} 


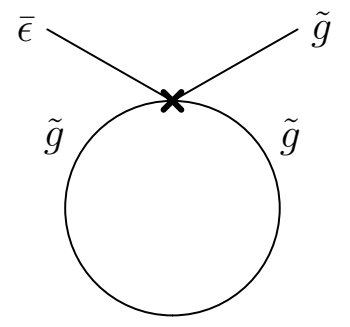

Topology (a)

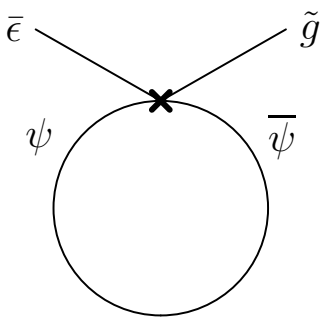

Topology (b)

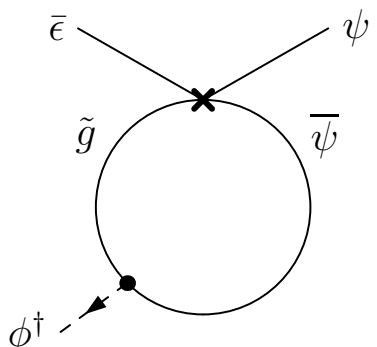

Topology (c)

Figure 1: Basic topologies of diagrams involving an insertion of $S\left(\Gamma_{\mathrm{cl}}\right)$, eq. (4.4). Only fermion lines are drawn; additional boson lines have to be attached in the actual diagrams. In the text, the $\gamma$-string attached to the second external fermion line is denoted as $A$, the $\gamma$-string attached to the closed fermion loop as $B$.

On the regularized level, the identities (4.6), (4.7), (4.8) are not necessarily satisfied. The right-hand side adds up to an expression that can be computed using the quantum action principle in the form (4.3). For example, for the first identity we obtain

$$
\Gamma_{\psi \bar{\epsilon} Y_{\phi_{i}}}^{\mathrm{DRED}} \Gamma_{\phi^{\dagger} \phi_{i}}^{\mathrm{DRED}}-\Gamma_{\phi^{\dagger} Y_{\psi_{i}} \bar{\epsilon}}^{\mathrm{DRED}} \Gamma_{\psi \bar{\psi}_{i}}^{\mathrm{DRED}}=i\left(\left[S\left(\Gamma_{\mathrm{cl}}\right)\right] \cdot \Gamma^{\mathrm{DRED}}\right)_{\phi^{\dagger} \psi \bar{\epsilon}}
$$

where the right-hand side denotes the 1PI Green function with external $\phi^{\dagger}, \psi, \bar{\epsilon}$ and an insertion of $S\left(\Gamma_{\mathrm{cl}}\right)$, eq. (4.4). Whether such Green functions vanish or constitute a supersymmetry breaking of DRED can often be determined quite easily by inspection of the corresponding Feynman diagrams.

All insertions in (4.4) are four-fermion operators, and the Green functions in our examples always have one external $\bar{\epsilon}$, a second external fermion line and one internal fermion loop. So all Feynman diagrams we have to consider involve one of the basic fermion topologies in Fig. 1 to which additional boson lines are attached. Topology (a) corresponds to an insertion of $\Delta_{\text {gauge }}$, and Topologies (b,c) to insertions of $\Delta_{\text {matter }}$ where either the gaugino or $\psi$ acts as the external fermion. Denoting the string of $\gamma$-matrices attached to the second external fermion line as $A$ and the $\gamma$ string associated to the closed fermion loop as $B$, the Feynman rules lead to the following general results:

$$
\begin{array}{ll}
\text { Topology (a): } & \gamma^{\mu}\left(B-B^{C}\right) \gamma_{\mu} A+\gamma^{\mu} A \operatorname{Tr}\left(\gamma_{\mu} B\right), \\
\text { Topology (b): } & 2\left(P_{L} B P_{R}-P_{R} B^{C} P_{L}\right) A-\gamma^{\mu} A \operatorname{Tr}\left(\gamma_{\mu} P_{L} B\right), \\
\text { Topology (c): } & \left(\gamma^{\mu} B \gamma_{\mu}-2 P_{R} B^{C}\right) P_{L} A-2 P_{L} A \operatorname{Tr}\left(P_{R} B\right) .
\end{array}
$$

All these expressions vanish identically in strictly four dimensions. In DRED and Q4S, however, it can be explicitly checked that the expressions only vanish if $B$ does not contain more than 4, 2, or $3 \gamma$-matrices in case (a), (b), or (c), respectively. E.g. 


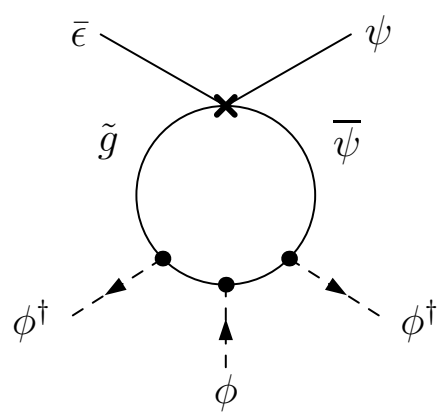

Figure 2: One-loop diagram corresponding to $\left(\left[S\left(\Gamma_{\mathrm{cl}}\right)\right] \cdot \Gamma^{\mathrm{DRED}}\right)_{\phi^{\dagger} \phi^{\dagger} \phi \psi \bar{\epsilon}}$.

if we set $B=\gamma^{\mu_{1}} \gamma^{\mu_{2}} \gamma^{\mu_{3}}$ in case (b) we obtain

$$
2 P_{L}\left(\gamma^{\mu_{1}} \gamma^{\mu_{2}} \gamma^{\mu_{3}}-\gamma^{\mu_{1}} g^{\mu_{2} \mu_{3}}+\gamma^{\mu_{2}} g^{\mu_{1} \mu_{3}}-\gamma^{\mu_{3}} g^{\mu_{1} \mu_{2}}\right)
$$

instead of zero, so this expression could lead to a violation of a Slavnov-Taylor identity if it appears in an actual diagram.

Now let us discuss the $\phi^{4}$-identity (4.8) at the one-loop level. Its possible violation is given by the diagram in Fig. 2 (+permutations of the boson lines). After integrating over the loop momentum, the $\gamma$-string $B$ can only contain the covariants $\not p_{1}, \not p_{2}, \not p_{3}$ with the three independent external momenta of the diagram. Hence after suitable simplifications $B$ cannot contain more than three $\gamma$-matrices, and this is not enough to lead to a non-vanishing contribution. This shows that identity (4.8) is valid in DRED at the one-loop level. By the same token, however, it is possible that (4.8) is violated at the two-loop level where e.g. the exchange of an additional vector boson can lead to more covariants within $B$.

Secondly we turn to identity (4.6) relating the $\phi$ and $\psi$ propagators. At the one-loop level, it has been verified already in [4] in various supersymmetric models by explicit evaluation of all Feynman diagrams corresponding to the Green functions in (4.6). The same verification is almost trivial if the quantum action principle is used: The only one-loop diagram contributing to the violation $\left(\left[S\left(\Gamma_{\mathrm{cl}}\right)\right] \cdot \Gamma^{\mathrm{DRED}}\right)_{\phi^{\dagger} \psi \bar{\epsilon}}$ is the "Topology (c)" diagram in Fig. 1. In this diagram, the $\gamma$-string $B$ contains at most two $\gamma$-matrices, and so the expression (4.10c) and thus the whole diagram vanishes.

At the two-loop level there are several diagrams; Fig. 3 shows one of them. After integrating over the fermion loop momentum and contracting all indices within the $\gamma$-string $B, B$ can only contain the covariants $\not p$, $\not k$, where $k$ is the second loop momentum, and $\gamma^{\mu}$ if $A$ and $B$ are connected by a vector boson as in Fig. 3. Hence $B$ can contain at most three $\gamma$-matrices if there is a virtual vector boson (which implies that the diagram is based on Topology (c)) and at most two $\gamma$-matrices in all other cases. Thus in all cases the respective expressions (4.10a), (4.10b), or (4.10d) vanish. This shows that the propagator identity (4.6) is valid in DRED even at the two-loop level. 


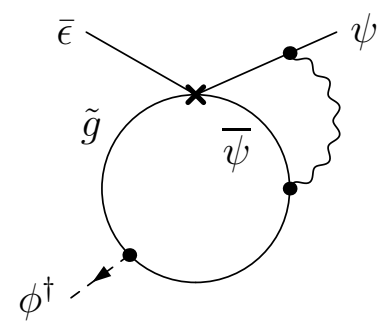

Figure 3: A two-loop diagram corresponding to $\left(\left[S\left(\Gamma_{\mathrm{cl}}\right)\right] \cdot \Gamma^{\mathrm{DRED}}\right)_{\phi^{\dagger} \psi \bar{\epsilon}}$. Such two-loop diagrams involving a virtual vector boson are the only ones where the $\gamma$-string $B$ can contain three $\gamma$-matrices after integration over the fermion loop momentum.
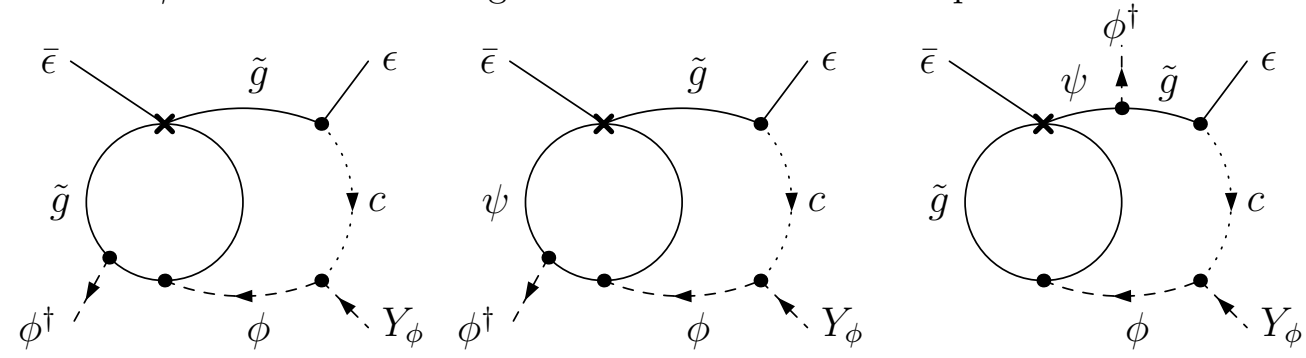

Figure 4: Two-loop diagrams corresponding to $\left(\left[S\left(\Gamma_{\mathrm{cl}}\right)\right] \cdot \Gamma^{\mathrm{DRED}}\right)_{\phi \epsilon \bar{\epsilon} Y_{\phi}}$.

Similar arguments can be applied to identity (4.7) for the supersymmetry transformations. There is no one-loop diagram contributing to $\left(\left[S\left(\Gamma_{\mathrm{cl}}\right)\right] \cdot \Gamma^{\mathrm{DRED}}\right)_{\phi \epsilon \bar{\epsilon} Y_{\phi}}$, which immediately proves that (4.7) is valid in DRED at the one-loop level. The two-loop diagrams are shown in Fig. 4 . After integrating over the fermion loop momentum, the $\gamma$-string $B$ can only contain the covariants $\not p$ and $\not k$, and thus $B$ cannot contain more than two $\gamma$-matrices. Thus again in all cases the respective expressions (4.10a), (4.10b), or (4.10c) vanish, and (4.7) holds in DRED at the two-loop level.

\section{Conclusions}

In this paper we have tried to put DRED on a firm mathematical basis by explicitly constructing the formally $D$ - and 4-dimensional objects and by establishing the quantum action principle. If the calculational rules of Section 2 are applied, no internal contradictions can appear. The quantum action principle provides an elegant all-order relation between the symmetry-properties of the Lagrangian $\delta_{\text {SUSY }} \mathcal{L}$ and Ward or Slavnov-Taylor identities.

Using this relation we have studied the supersymmetry-properties of DRED. If a totally anticommuting $\gamma_{5}$ is used as traditionally done in DRED, the breaking term (4.4) consists only of four-fermion operators that would vanish if Fierz identities were valid. We have shown that there are simple rules as to when these expressions can be non-vanishing, depending only on the number of $\gamma$-matrices in the closed fermion line. 
These rules can be easily applied to study the breaking of Slavnov-Taylor identities; hence by using the quantum action principle many more identities are amenable to checks, and the checks themselves are drastically simplified.

Here we considered the identities for propagators and supersymmetry transformations of matter fields. It was straightforward to show that these identities are valid up to the two-loop level in DRED. In addition, we verified the identity for the $\phi^{4}$-interaction, which had not been considered in the literature before, at the one-loop level. The rules for the insertions indicate that at higher orders supersymmetry relations could be violated. In that case, supersymmetry-restoring counterterms might be necessary in DRED similar to DREG, but to a much smaller extent.

It will be an important future task to delineate the precise range of validity of the supersymmetry relations in DRED and to find the necessary counterterms. Furthermore, it would be desirable to include soft supersymmetry breaking into the analysis along the lines of Refs. [7]. However, given our examples it seems plausible that for many foreseeable calculations up to the two-loop level DRED can be used without having to worry about breaking of supersymmetry.

The treatment of $\gamma_{5}$ still causes problems. Like naive DREG, DRED with totally anticommuting $\gamma_{5}$ necessarily implies that traces involving $\gamma_{5}$ and an arbitrary number of $\gamma^{\mu}$ 's are zero. Hence e.g. fermion triangle diagrams with an axial vector current cannot be correctly computed in this scheme. The alternative definition of a strictly 4-dimensional $\gamma_{5}$ avoids this defect, but like in the HVBM-scheme of DREG, supersymmetry and gauge invariance are broken already at one-loop order.

However, in any given calculation it is usually easy to see whether this problem is relevant, and very often it is not (see e.g. Ref. [24] for the discussion of an example at the two-loop level). Moreover, for the $\gamma_{5}$-problem in DREG there are elaborate practical prescriptions, e.g. in Refs. [25]. Using the present results as a basis one can hope to find similar prescriptions for supersymmetric theories within DRED that yield correct results for traces but allow the use of the anticommuting $\gamma_{5}$ and avoid breaking of supersymmetry and gauge invariance as much as possible.

\section{Acknowledgements}

I thank the participants of the SPA-workshop on DRED at DESY, January 2005, where this work was begun, for stimulating discussions. In particular I am grateful to W. Hollik, I. Jack, P. Zerwas, and M. Gorbahn for many discussions, questions and comments.

\section{A. Explicit construction of Q4S}

In this Appendix we give an explicit construction of the objects introduced in Sec. 2 and show that they satisfy all desired relations. First, the momentum integral is obviously identical in DRED and DREG. In [16] it has been shown that a formally 
$d$-dimensional integral of functions of the form $f\left(p^{2}, p q_{1}, \ldots p q_{n}\right)$ can be defined if the underlying vector space is infinite dimensional. $d$-dimensionality of the integral is expressed in terms of the scaling and normalization properties

$$
\int d^{d} p f(s p)=s^{-d} \int d^{d} p f(p), \quad \quad \int d^{d} p e^{-p^{2} / 2}=\pi^{d / 2} .
$$

While the contravariant metric tensor $g^{\mu \nu}$ on this infinite dimensional vector space is defined by its components, its covariant counterpart $g_{\mu \nu}$ is defined as a bilinear form on this space as

$$
g_{\mu \nu} a^{\mu \nu} \equiv \frac{d \Gamma(d / 2)}{\pi^{d / 2}} \int d^{d} p p_{\mu} p_{\nu} a^{\mu \nu} \delta\left(p^{2}-1\right) .
$$

With this definition one has the formally $d$-dimensional result $g_{\mu \nu} g^{\mu \nu}=d$. Further properties of the $d$-dimensional integral and metric tensor are discussed in [17].

In DRED, we simply perform the construction of the $d$-dimensional integral and metric tensor twice: once with $d=D$ (and metric signature $+-\ldots$ ), and once with $d=\epsilon=4-D$ (and signature $---\ldots$ ). This yields two infinite dimensional vector spaces $\mathrm{Q} D \mathrm{~S}$ and $\mathrm{Q} \epsilon \mathrm{S}$ and two metric tensors $\hat{g}^{\mu \nu}$ and $\tilde{g}^{\mu \nu}$ with $\hat{g}_{\mu \nu} \hat{g}^{\mu \nu}=D$, $\tilde{g}_{\mu \nu} \tilde{g}^{\mu \nu}=\epsilon$. The $D$-dimensional integral in the first of these spaces constitutes the momentum integral in DRED, the $\epsilon$-dimensional integral of $\mathrm{Q} \epsilon \mathrm{S}$ has no other purpose than defining $\tilde{g}_{\mu \nu}$. We can then construct the quasi-4-dimensional space Q4S as the direct sum $\mathrm{Q} 4 \mathrm{~S}=\mathrm{Q} D \mathrm{~S} \oplus \mathrm{Q} \epsilon \mathrm{S}$ and define its metric tensor as $g^{\mu \nu} \equiv \hat{g}^{\mu \nu}+\tilde{g}^{\mu \nu}{ }^{3}$ Then all equations (2.3) are satisfied by construction.

In order to define $\gamma$-matrices in Q4S, we rely on the existence of $\gamma$-matrices in DREG, in particular on the the explicit representation of Ref. [17]. Denoting these matrices as $\Gamma^{\mu}(\mu=0,1,2 \ldots \infty)$, they satisfy $\left\{\Gamma^{\mu}, \Gamma^{\nu}\right\}=2 g^{\mu \nu}, \Gamma^{\mu \dagger}=\Gamma^{0} \Gamma^{\mu} \Gamma^{0}$, and after suitable reordering $\Gamma^{\mu *}=(-1)^{\mu} \Gamma^{\mu}$. In DRED, according to the structure of Q4S as the sum of two vector spaces, the vector of $\gamma$-matrices has the decomposition $\gamma=\left(\begin{array}{l}\hat{\gamma} \\ \tilde{\gamma}\end{array}\right)$ or $\gamma^{\mu}=\hat{\gamma}^{\mu}+\tilde{\gamma}^{\mu}$. We define

$$
\begin{array}{rlrl}
\hat{\gamma}^{0} & =\left(\begin{array}{cc}
\Gamma^{0} & 0 \\
0 & \Gamma^{0}
\end{array}\right), & \hat{\gamma}^{i}=\left(\begin{array}{cc}
\Gamma^{2 i+4} & 0 \\
0 & \Gamma^{2 i+4}
\end{array}\right) \quad(i \geq 1), \\
\tilde{\gamma}^{\mu}=\left(\begin{array}{cc}
0 & -i \Gamma^{2 \mu+5} \\
i \Gamma^{2 \mu+5} & 0
\end{array}\right) & (\mu \geq 0), & \\
\gamma_{5}=\left(\begin{array}{cc}
0 & \Gamma^{4} \\
-\Gamma^{4} & 0
\end{array}\right), & C=\left(\begin{array}{cc}
0 & -\Gamma^{2} \Gamma^{0} \\
\Gamma^{2} \Gamma^{0} & 0
\end{array}\right) .
\end{array}
$$

\footnotetext{
${ }^{3}$ Any vector $p$ in $\mathrm{Q} 4 \mathrm{~S}$ has the decomposition $p=\left(\begin{array}{l}\hat{p} \\ \tilde{p}\end{array}\right)$, where $\hat{p} \in \mathrm{Q} D \mathrm{~S}, \tilde{p} \in \mathrm{Q} \in \mathrm{S}$. We can naturally identify $\hat{p} \equiv\left(\begin{array}{c}\hat{p} \\ 0\end{array}\right)$ and $\tilde{p} \equiv\left(\begin{array}{c}0 \\ \tilde{p}\end{array}\right)$ and write $p^{\mu}=\hat{p}^{\mu}+\tilde{p}^{\mu}$. Similarly, the relation $g^{\mu \nu} \equiv \hat{g}^{\mu \nu}+\tilde{g}^{\mu \nu}$ really means the decomposition $g \equiv\left(\begin{array}{ll}\hat{g} & 0 \\ 0 & \tilde{g}\end{array}\right)$. Strictly speaking, the indices $\mu$ in Q4S can be considered as pairs $\mu=(a ; i)$, where $a=1,2$ and $i=0,1,2,3, \ldots$ such that $p^{(1 ; i)}=\hat{p}^{i}, p^{(2 ; i)}=\tilde{p}^{i}$.
} 
One can immediately verify that this definition satisfies all properties listed in Sec. 2, and in addition we can read off the properties

$$
\begin{aligned}
& \gamma^{\mu *}=\gamma^{\mu}, \quad \gamma_{5}^{*}=\gamma_{5}, \quad C^{*}=C, \\
& C^{2}=-1, \quad\left\{C, \gamma^{0}\right\}=0, \quad C^{\dagger}=-C,
\end{aligned}
$$

which implies in particular $\left(\psi^{C}\right)^{C}=\psi$ for any spinor.

Traces of a product $A$ of $\gamma$-matrices can be defined by [17]

$$
\operatorname{Tr} A \equiv \lim _{N \rightarrow \infty} \frac{4}{N} \sum_{j=1}^{N} A_{j j},
$$

so that in particular $\operatorname{Tr} 1=4$. Since all traces of $\gamma$-matrices can be reduced to $\operatorname{Tr} 1$ times metric tensors by applying the anticommutation relations, results of traces are formally the same in four dimensions and in $\mathrm{Q} 4 \mathrm{~S}$ as long as no $\gamma_{5}$ is present.

\section{References}

[1] G. 't Hooft and M. Veltman, Nucl. Phys. B 44 (1972) 189.

[2] W. Siegel, Phys. Lett. B 84 (1979) 193.

[3] W. Beenakker, R. Höpker and P. M. Zerwas, Phys. Lett. B 378 (1996) 159 [arXiv:hep-ph/9602378].

[4] W. Hollik, E. Kraus and D. Stöckinger, Eur. Phys. J. C 11 (1999) 365 [arXiv:hep-ph/9907393];

W. Hollik and D. Stöckinger, Eur. Phys. J. C 20 (2001) 105 [arXiv:hep-ph/0103009];

I. Fischer, W. Hollik, M. Roth and D. Stöckinger, Phys. Rev. D 69 (2004) 015004 [arXiv:hep-ph/0310191].

[5] P. L. White, Class. Quant. Grav. 9 (1992) 1663.

[6] N. Maggiore, O. Piguet and S. Wolf, Nucl. Phys. B 458 (1996) 403 [Erratum-ibid. B 469 (1996) 513] [arXiv:hep-th/9507045], Nucl. Phys. B 476 (1996) 329 [arXiv:hep-th/9604002].

[7] W. Hollik, E. Kraus and D. Stöckinger, Eur. Phys. J. C 23 (2002) 735 [arXiv:hep-ph/0007134];

W. Hollik, E. Kraus, M. Roth, C. Rupp, K. Sibold and D. Stöckinger, Nucl. Phys. B 639 (2002) 3 [arXiv:hep-ph/0204350].

[8] W. Siegel, Phys. Lett. B 94 (1980) 37.

[9] S. Heinemeyer, W. Hollik and G. Weiglein, arXiv:hep-ph/0412214. 
[10] P. M. Zerwas, Talk given at the ECFA "Physics and Detectors for a Linear Collider" Workshop, Durham 1 - 4 September 2004;

http://www.ippp.dur.ac.uk/ECFA04/program.html.

[11] W. Beenakker, H. Kuijf, W. L. van Neerven and J. Smith, Phys. Rev. D 40 (1989) 54 .

[12] J. Smith and W. L. van Neerven, arXiv:hep-ph/0411357.

[13] I. Jack, D. R. T. Jones and A. F. Kord, Phys. Lett. B 579, 180 (2004) [arXiv:hep-ph/0308231].

[14] I. Jack and D. R. T. Jones, "Regularisation of supersymmetric theories", in Kane, G.L. (ed.): Perspectives on supersymmetry 149-167; [arXiv:hep-ph/9707278].

[15] L. V. Avdeev, G. A. Chochia and A. A. Vladimirov, Phys. Lett. B 105 (1981) 272; L. V. Avdeev and A. A. Vladimirov, Nucl. Phys. B 219 (1983) 262.

[16] K. G. Wilson, Phys. Rev. D 7 (1973) 2911.

[17] J. Collins, "Renormalization", Cambridge Monographs on Mathematical Physics.

[18] P. Breitenlohner and D. Maison, Commun. Math. Phys. 52 (1977) 11.

[19] J. H. Lowenstein, Phys. Rev. D 4 (1971) 2281, Commun. Math. Phys. 24 (1971) 1; Y. M. Lam, Phys. Rev. D 6 (1972) 2145; Phys. Rev. D 7 (1973) 2943.

[20] D. M. Capper, D. R. T. Jones and P. van Nieuwenhuizen, Nucl. Phys. B 167 (1980) 479 .

[21] P. Breitenlohner and D. Maison, "Renormalization Of Supersymmetric Yang-Mills Theories," in Cambridge 1985, Proceedings, Supersymmetry and Its Applications, 309-327.

[22] S. P. Martin and M. T. Vaughn, Phys. Lett. B 318 (1993) 331 [arXiv:hep-ph/9308222];

I. Jack, D. R. T. Jones and C. G. North, Nucl. Phys. B 473 (1996) 308 [arXiv:hep-ph/9603386], Phys. Lett. B 386 (1996) 138 [arXiv:hep-ph/9606323], Nucl. Phys. B 486 (1997) 479 [arXiv:hep-ph/9609325].

[23] C. Rupp, R. Scharf and K. Sibold, Nucl. Phys. B 612 (2001) 313 [arXiv:hep-th/0101165], [arXiv:hep-th/0101153].

[24] S. Heinemeyer, D. Stöckinger and G. Weiglein, Nucl. Phys. B 699 (2004) 103 [arXiv:hep-ph/0405255]. D. Stöckinger, Nucl. Phys. Proc. Suppl. 135 (2004) 311 [arXiv:hep-ph/0406306].

[25] S. A. Larin, Phys. Lett. B 303 (1993) 113 [arXiv:hep-ph/9302240]; T. L. Trueman, Z. Phys. C 69 (1996) 525 [arXiv:hep-ph/9504315]. 